Solid State Communications, Vol.31, pp.465-468.

Pergamon Press Ltd. 1979. Printed in Great Britain.

\title{
DETERMINATION OF LANDAU LEVEL LIFETIMES IN n-GaAs
}

\section{H.J.A. Bluyssen, J.C. Maan and P. Wyder}

Research Institute for Materials, University of Nijmegen, Toernooiveld, Nijmegen, The Netherlands

(Received 4 May 1979 by A.R. Miedema)

\begin{abstract}
The lifetime of electrons in the first excited Landau level of n-GaAs is determined from a combination of measurements of far infrared cyclotron resonance induced absorption and conductivity change. Values of $\mathrm{T}_{1}$ of the order of $10^{-8} \mathrm{~s}$ for densities of excited electrons of $10^{11} \mathrm{~cm}^{-3}$ and a temperature dependence of $\mathrm{T}-2.7$ are found. An upper limit for the $\mathrm{N}=0$ Landau level to donor recombination time of the order of $10^{-9} \mathrm{~s}$ was derived from pulsed conductivity measurements.
\end{abstract}

Recently two different methods have been reported ${ }^{1}, 2$ to determine the lifetime of electrons in the $\mathrm{N}=1$ Landau level in $\mathrm{n}-\mathrm{InSb}$ by cyclotron resonance absorption. Gornik et al. ${ }^{1}$ determined this lifetime from saturation absorption measurements with an optically pumped molecular laser. Müller and Gornik ${ }^{2}$ used observations of the transient decay of the photoconductivity following pulsed far infrared excitation.

In this paper a new method is presented to determine the Landau level lifetime from a combination of measurements of the far infrared cyclotron resonance induced absorption and conductivity change in $n$-GaAs. The observed lifetimes which are of the order of $10^{-8} \mathrm{~s}$ for electron densities in the excited level of $10^{11} \mathrm{~cm}^{-3}$ suggest acoustic phonon scattering as the dominant relexation process and are consistent with upper limits determined from pulsed excitation measurements. The lifetime shows further a temperature dependence of $\mathrm{T}-2.7$ for temperature values of $\mathrm{T}=11 \mathrm{~K}-34 \mathrm{~K}$. From pulsed cyclotron resonance induced conductivity measurements an upper limit of the order of $10-9 \mathrm{~s}$ for the Iifetime of the $\mathrm{N}=0$ Landau level i.e. the recombination-time to the donor ground state is derived.

As shown in a previous paper ${ }^{3}$ the mechanism underlying the change in the conductivity under cyclotron resonance conditions can be described by a three level model (see Fig. 1). Fxcitation of electrons from the lowest Landau level (2) to the first excited Landau level ( 3 ) is followed by thermal equilibration of electrons from the donor ground state (1). to the depleted lowest Landau level, thus increasing the free carrier density $(\Delta n)$ and therefore the conductivity. This process can be described by the following set of rate equations:

$\mathrm{dn}_{1} / \mathrm{dt}=-\mathrm{t}_{12}^{-1} \mathrm{n}_{1}+\mathrm{t}_{21}^{-1} \mathrm{n}_{2}$

$a n_{2} / d t=t_{12}^{-1} n_{1}-t_{21}^{-1} n_{2}+t_{32}^{-1} n_{3}-w\left(n_{2}-n_{3}\right)$

$\mathrm{dn}_{3} / \mathrm{dt}=\mathrm{w}\left(\mathrm{n}_{2}-\mathrm{n}_{3}\right)-\mathrm{t}_{32}^{-1} \mathrm{n}_{3}-\mathrm{t}_{31}^{-1} \mathrm{n}_{3}$ where $W$ denotes the induced transition rate of cyclotron resonant absorption. The electrons which are excited to the $\mathbb{N}=1$ Landau level relax to the $\mathbb{N}=0$ Landau state and the donor ground state with the transition rates $t-1$ and $\left.t_{\overline{3}}\right]$ respectively. The electrons occupying the lowest Landau state return to the donor ground state with the recombination rate $t_{\overline{2}} 1$ and the reverse process occurs with $t_{12}^{-1}$. It is further assumed that $t \overline{3} \mid \ll t_{\overline{3}} \frac{1}{2}$ and that at thermal equilibrium $n \mathcal{\beta}=0$.

From the steady state condition for the rate equations, i.e. $\mathrm{dn}_{j} / \mathrm{dt}=0$, the conservation of electrons $n_{1}+n_{2}+n_{3}=n 9+n 8$, and the detailed balance at thermal equilibrium, $t_{12}^{-1} n_{1}^{0}=t_{2}^{-1} \mid n_{2}$, one can show that the absorption coefficient $\alpha$ is given by the following expression:

$\alpha \equiv\left(n_{2}-n_{3}\right) \frac{W \hbar \omega}{I}=\frac{\hbar \omega}{I} \frac{W}{1 \mp W T} n_{2}^{\circ}$

where $I$ is the intensity of radiation at the sample, and

$T=\left(2 t_{12}^{-1}+t_{21}^{-1}\right) / t_{32}^{-1}\left(t_{12}^{-1}+t_{21}^{-1}\right)$

For the relative change in the conductivity one obtains,

$$
\begin{aligned}
\Delta \sigma / \sigma & =\Delta \mathrm{n} / \mathrm{n}_{2}^{\circ}=\left(\mathrm{n}_{2}+\mathrm{n}_{3}-\mathrm{n}_{2}^{0}\right) / \mathrm{n}_{2}^{\circ} \\
& =\frac{\mathrm{W}}{1+\mathrm{WT}} \mathrm{T}_{1} \frac{\frac{\mathrm{n}}{1}}{\mathrm{n}_{1}^{\circ}+\mathrm{n}_{2}^{\circ}}
\end{aligned}
$$

with $T_{1}=t_{32}$. The electronic lifetime $T_{1}$ follows directly from Eq. (2), (3) and

(4)

$\mathrm{T}_{1}=\frac{\mathrm{h} \omega}{\mathrm{I \alpha}} \frac{\Delta \sigma}{\sigma} \mathrm{n}_{2}^{\circ} \frac{\mathrm{n}_{1}^{\circ}+\mathrm{n}_{2}^{\circ}}{\mathrm{n}_{1}^{\circ}}$ 


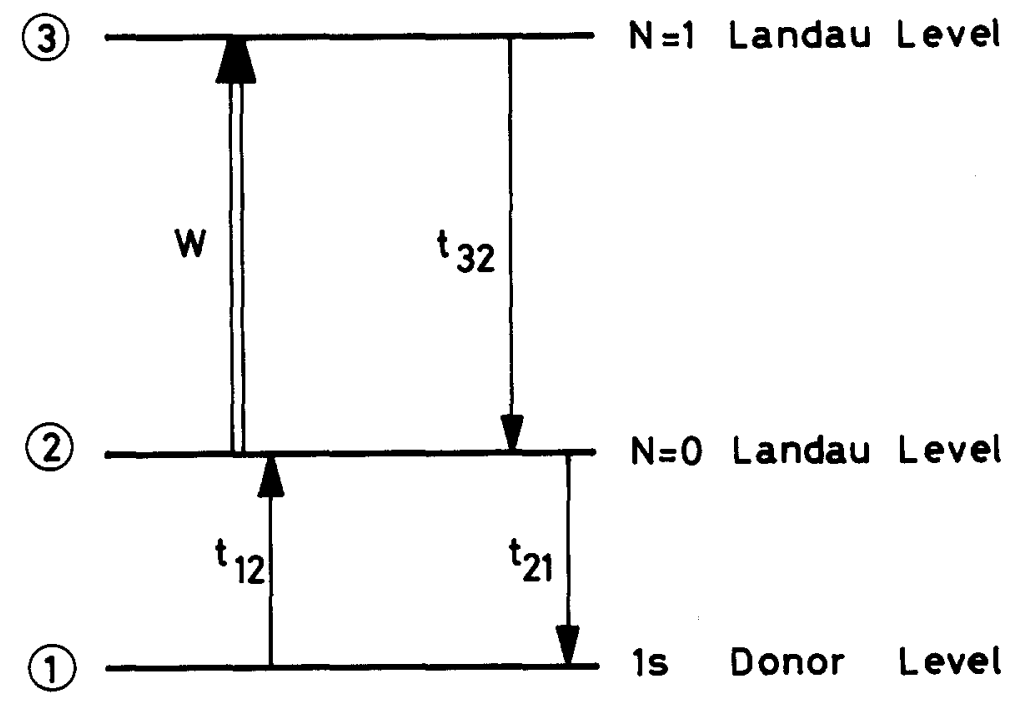

Fig. 1 : Energy level structure of the donor ground state (1) and the two lowest conduction band states (2) and (3) in a magnetic field. W denotes the radiation induced transition rate; $t_{12}^{-1}, t_{21}^{-1}$ and $t_{32}^{-1}$ describe the relaxation rates between the different levels.

showing that $T_{1}$ can be calculated from the relative change in the conductivity $\Delta \sigma / \sigma$, the absorption coefficient $\alpha$, the carrier density $\mathrm{n}_{2}$ and the number of unionized donors $n \oint=N_{D}-N_{A}-n g$ at thermal equilibrium. $\Delta \sigma / \sigma$ was measured as a relative change in the voltage over the sample under constant current conditions; $\mathrm{n}_{2}^{\circ}$ was determined from Hall-effect measurements as a function of temperature. The peak absorption coefficient $\alpha$ was calculated from the transmission data by using $\alpha=\left[\ln \left(W^{\prime} / W^{\prime \prime}\right)+\left(r W^{\prime \prime} / W^{\prime}\right)^{2}-r^{2}\right] / \alpha$, where $r$ is the reflectance of each sample surface, $d$ is the sample thickness and $W^{\prime}$ and $W^{\prime \prime}$ are the intensities off and on resonance. The samples were chosen such that $\alpha d<1$ to minimize errors due to variation of the absorbed power within the sample.

Fig. 2 shows the values of $T_{1}$ as a function of the temperature deduced from measurements on an n-GaAs sample of the following characteristics: $n=1.6 \times 1015 \mathrm{~cm}-3$, $\mu=7610 \mathrm{~cm} 2 \mathrm{~V}^{-1} \mathrm{~s}^{-1}$ at $\mathrm{T}=300 \mathrm{~K}$. Both the transmission and the conductivity were measured in the Faraday configuration in the temperature range from $\mathrm{T}=11 \mathrm{~K}$ to $34 \mathrm{~K}$ and with far infrared radiation from a cw optically pumped $\mathrm{CH}_{3} \mathrm{OH}$ laser. The output power of the $\mathrm{CH}_{3} \mathrm{OH}$ laser of $10 \mathrm{~mW}$ at $\lambda=118.8 \mu \mathrm{m}$ was attenuated to about $10 \mu \mathrm{W}$ at the sample. The change in the transmission of the radiation due to the cyclotron resonant absorption was detected by a liquid helium cooled si-bolometer. The calculated values of $T_{1}$ vary from $5 \times 10^{-8}$ at $11 \mathrm{~K}$ to $2 \cdot 10^{-9} \mathrm{~s}$ at $34 \mathrm{~K}$ for numbers of excited carriers from $n_{3}=1.1 \mathrm{x}$ $10^{11} \mathrm{~cm}^{-3}$ to $\mathrm{n}_{3}=0.12 \times 10^{11} \mathrm{~cm}^{-3}$ respectively and show a temperature dependence of $\mathrm{T}-2.7$
(See Fig. 2 ). The magnitude of the observed lifetime and its temperature dependence suggest acoustic phonon scattering as the dominant relaxation process $2,4,5$.

From the steady state analysis of the three level model it follows that the lifetimes cannot be obtained from steady state measurements. In order to get at least some quantitative experimental confirmation about these lifetimes we also measured the conductivity response of the $n$-GaAs sample to a far infrared pulse under cyclotron resonance conditions. The far infrared pulses at $\lambda=118.8 \mu \mathrm{m}$ were generated in a $\mathrm{CH}_{3} \mathrm{OH}$ molecular laser optically pumped by a simultaneously current- and Q-switched conventional $\mathrm{CO}_{2}$ laser. A peak power of about $10 \mathrm{~kW}$ and a pulse duration of $100 \mathrm{~ns}$ were obtained for the $\mathrm{P}(36)$ pumpline giving rise to very strong far infrared pulses up to pressures of $\mathrm{CH}_{3} \mathrm{OH}$ of a few Torr. A more complete description of the system will be given elsewhere 6 . The electrical scheme for detection of the pulsed conductivity change in the n-GaAs sample was similar to that used by Kohl et al. 7 for their fast far infrared photoconductive detector and had a risetime of 2 ns.

A typical result is presented in Fig. 3 which shows the time dependency of the photoconductive response of the sample to a far infrared pulse at the cyclotron resonance field. The observed risetime of the pulse of $25 \mathrm{~ns}$ gives an upper limit to the generation rate $t_{1} 1$ as follows from the three level model of Fig. ' From detailed balance at thermal equilibrium, i.e. $\left.t_{1}-1 n_{1}=t_{2}\right\} n_{2}^{\circ}$, and the value of $t_{12}<2.10^{-8}$ s one obtains for the lifetime of the electrons in the $\mathrm{N}=0$ Landau level an 


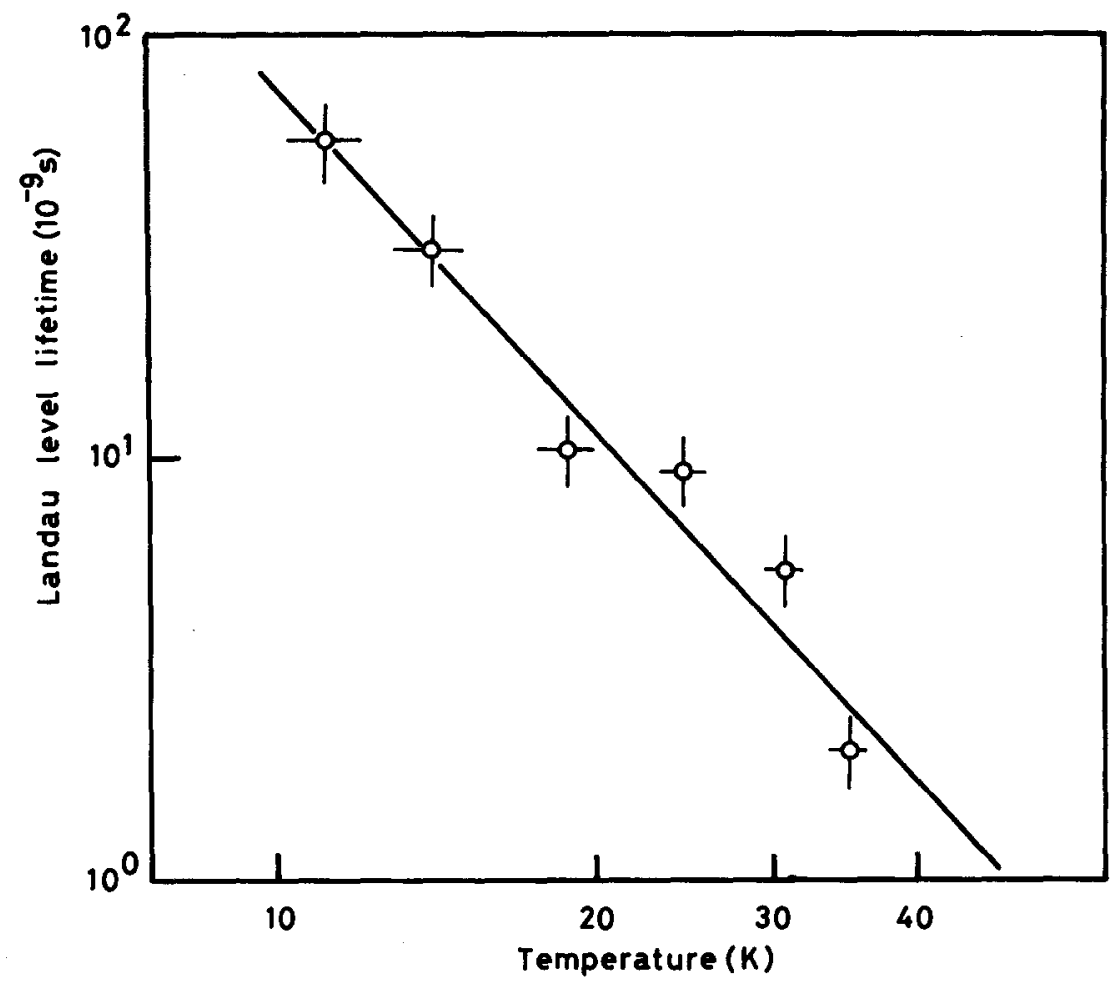

Fig. 2 : Temperature dependence of the electronic lifetime of the first excited Landau level in $n$-GaAs at $6.39 \mathrm{~T}$. The solid line presents the fit to the data using $\log \mathrm{T}_{1}=-4.4$ $( \pm 1.2)-2.72( \pm 0.4) \log \mathrm{T}$. The absolute values for the time are subject to a systematic error in the estimation of the incident far infrared power of about $50 \%$.

upper limit of $t_{21}<2.10-9 \mathrm{~s}$ at $\mathrm{T}=12 \mathrm{~K}$. These values are consistent with the ones obtained by Melngailis et al. ${ }^{8}$ from recombinative radiation measurements and by Stillman et al.9 from the recombination time of impact ionized free carriers for zero magnetic field. The decay time of the observed pulse of $125 \mathrm{~ns}$ gives an upper limit to the effective recombination time resulting from the cascade transition $\mathrm{N}=1$ to $N=0$ t.o donor ground state. Since $t_{21}<10-9 s$ it follows that $t_{32}<10^{-7}$ s which is not inconsistent with the results of the steady state measurements as described above.

In conclusion we have shown that a combination of measurements of the absorption coefficient and the associated conductivity change under cyclotron resonance conditions can be used to determine the electronic lifetime of the first excited Landau level in n-GaAs. Though this method has been used for temperatures from $11 \mathrm{~K}$ to $34 \mathrm{~K}$ this region can be easily extended to lower temperatures. To higher temperatures the method is limited by the condition for Landau quantization $\hbar \omega_{c}=\hbar \mathrm{eB} / \mathrm{m}^{3 \mathrm{H}}>\mathrm{kT}$. A principal limitation arises from the donor ionization energy since for $\mathrm{E}_{\mathrm{D}}<\mathrm{kT}$ the donors are completely ionized and $\Delta \sigma / \sigma \approx 0$.
This method has substantial advantages over saturation absorption measurements and does not have the experimental complexity of the pulsed method. In fact saturation absorption measurements are only feasible for determination of lifetimes in highly nonparabolic materials like In-Sb, where absorption occurs only between two Landau levels. In case of the nearly parabolic conduction band of $n-G a A s$ the Landau levels are nearly equally spaced so that saturation absorption implies continuous excitation in the Landau ladder up to the optical phonon emission energy, $\mathrm{E}_{\mathrm{Op}} \approx 35 \mathrm{meV}$. Lifetimes extracted from saturation absorption measurements in a parabolic material will therefore be determined by optical phonon emission. Moreover the large number of excited carriers which are present at saturating conditions changes the relaxation mechanism from acoustical phonon- to electron-electron scattering as was shown by Gornik et al. for $n$-InSb. The method described in this paper allows determination of electronic lifetimes at nearly thermal equilibrium conditions as was shown. The method can easily be extended to considerable deviations from thermal equilibrium by using higher intensities of the incident radiation thus allowing observations re- 


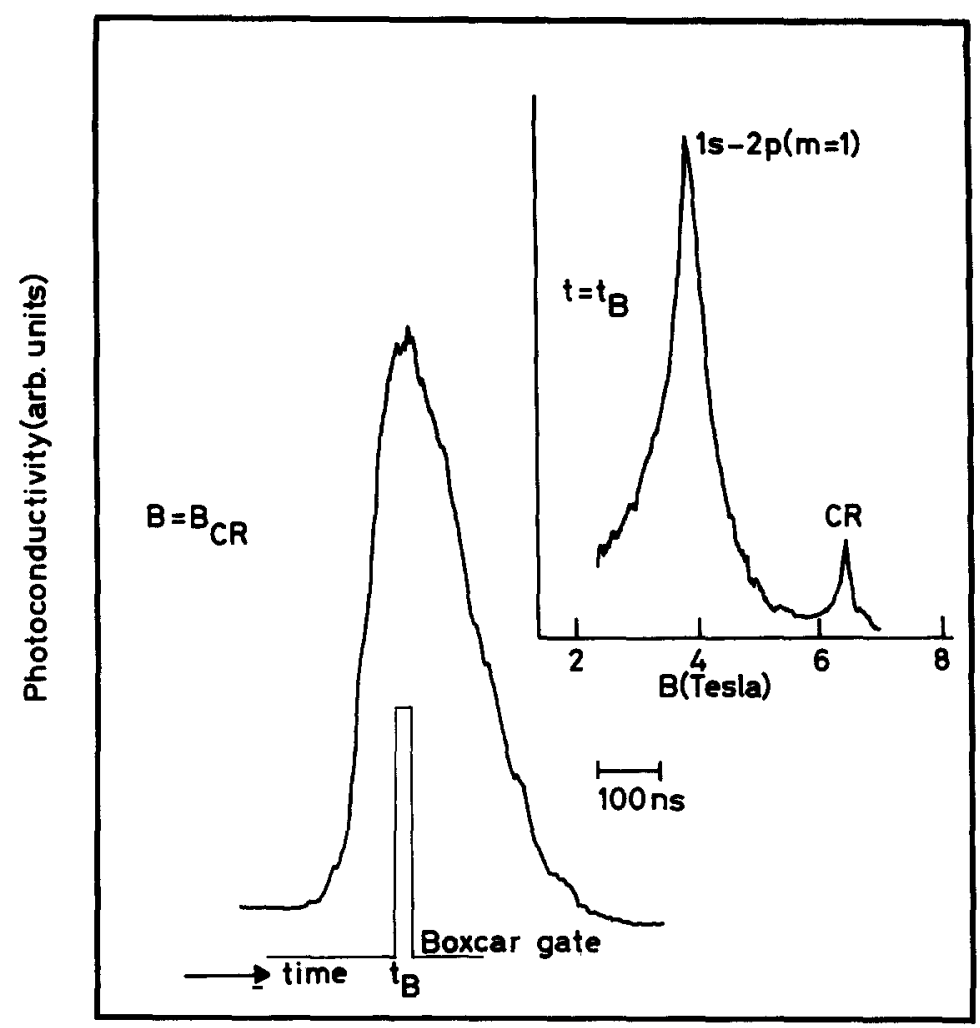

Fig. 3 : Pulse shape (wavelength $\lambda: 118.8 \mu \mathrm{m}$ ) of the photoconductive response of a $n$-GaAs sample. The pulse shape is independent of the magnetic field; the peak amplitude varies with magnetic field as is shown in the insert. The pulse shape gives an upper limit (determined by the measuring system) for the relevant electronic lifetimes.

lated to the hot electron effect as mentioned above.

Acknowledgement - We are pleased to thank A. van Etteger and B. Tan for their valuable contributions to the experimental part of this work and C.P. Damen of the Philips' Research

\begin{abstract}
Laboratories for preparing the samples. Part of this work has been supported by the "Stichting voor Fundamenteel Onderzoek der Materie" (FOM) with financial support of the "Nederlandse Organisatie voor Zuiver Wetenschappelijk Onderzoek" (ZWO).
\end{abstract}

1. GORNIK, E., CHANG, T.Y., BRIDGES, T.J., NGUYEN, V.T., MCGEE, J.D., Phys. Rev. Lett. 40 , 1151 (1978).

2. MULLER, W., GORNIK, E., Solid State Electronics 21, 1455 (1978).

3. BLUYSSEN, H.J.A., MAAN, J.C., VAN RUYYEN, L.J., WILLIAMS, F., WYDER, P., Solid State Commun. 25, 895 (1978).

4. PUTLEY, E.H., Phys. Stat. Sol. 6,571 (1964).

5. PARTE, H., MULLER, W., KOHL, F., GORNIK, E., J. Phys. C: Solid State Phys. 11, 1091 (1978).

6. BLUYSSEN, H.J.A., VAN ETTEGER, A., MAAN, J.C., to be published,

7. KOHL, F., MULLER, W., GORNIK, E., Infrared Physics 18, 697 (1978).

8. MEINGAILIS, I., STILLMAN, G.E., DIMMOCK, J.O., WOLFF, C.M., Phys. Rev. Lett. 23, 1111 (1969).

9. STILIMAN, G.E., WOLFE, C.M., MELNGAILIS, I., PARKER, C.D., TANNENWALD, P.E., DIMMOCK, J.O., Appl. Phys. Lett. 13, 83 (1968). 